www.nature.com/ejhg

\title{
Testing for BRCA1 mutations: a cost-effectiveness analysis
}

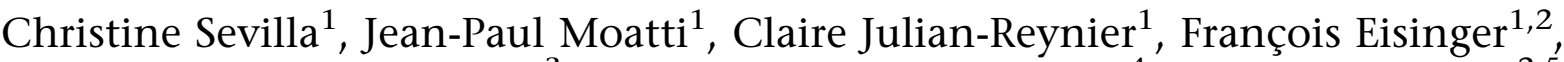 \\ Dominique Stoppa-Lyonnet ${ }^{3}$, Brigitte Bressac-de Paillerets ${ }^{4}$ and Hagay Sobol ${ }^{*, 2,5}$
}

\author{
${ }^{1}$ INSERM U379, Paoli-Calmettes Institute, Marseilles, France; ${ }^{2}$ Department of Genetic Oncology-INSERM EPI9939, \\ Paoli-Calmettes Institute, Marseilles, France; ${ }^{3}$ Department of Genetic Oncology, Curie Institute, Paris, France; \\ ${ }^{4}$ Department of Molecular Genetics, Gustave Roussy Institute, Villejuif, France; ${ }^{5}$ Faculté de Médecine, Université de la \\ Méditerranée, Marseilles, France
}

Breast carcinoma is the most common type of cancer affecting women in the Western world. The hereditary forms, which amount from 5 to $10 \%$ of all the cases of breast cancer, mainly involve BRCA1 or BRCA2 mutations. Due to the diagnostic strategy used by the patent owner, Direct DNA sequencing (DS) may become the only BRCA1/2 test procedure available, although there exist several alternative strategies. A cost-effectiveness study was carried out using BRCA1 testing as a model. The main techniques available for performing mutation searches were assessed: DS, denaturing high performance liquid chromatography (DHPLC), single-strand conformation polymorphism (SSCP), denaturing gradient gel electrophoresis (DGGE), heteroduplex analysis (HA), fluorescent assisted mismatch analysis (FAMA) and the protein truncation test (PTT). Twenty strategies involving the use of one or more techniques were then devised for performing the complete genetic analysis. DS was adopted as the 'gold standard' for effectiveness. All the strategies except for DS involved a two-step procedure. The first step consisted of pre-screening the 22 coding exons of BRCA1. The second step consisted of performing DS only on the variations detected in the coding sequence. The cost of the strategies tested, including a pre-screening stage, turned out to be 30 to $90 \%$ lower than that of DS, whatever annual use was made of the equipment. The most cost-effective strategy, ie, that corresponding to the lowest cost per mutation detected, was found to be a combination between PTT on exon 11 (60\% of the coding sequence) and HA on the remaining 21 exons $\left(\mathrm{PTT}_{11}+\mathrm{HA}_{21}\right)$. However, since a high false negative rate is associated with this strategy, at least four other strategies are worth mentioning: PTT $11+$ DHPLC $_{21}$, DHPLC alone, FAMA $11+^{+}$ DHPLC $_{21}$ and FAMA alone. Our results on genetic testing for breast cancer show that DS is not the most cost-effective method available. The monopolist approach of the firm which owns the patents on the BRCA1/2 genes, may, therefore limit the use of the most cost-effective strategies.

European Journal of Human Genetics (2002) 10, 599-606. doi:10.1038/sj.ejhg.5200854

Keywords: cost-effectiveness; BRCA1; BRCA2; genetic testing; breast cancer susceptibility

Introduction

Breast carcinoma is the most common type of cancer affecting women in the Western world. Hereditary Breast Cancer

\footnotetext{
*Correspondence: Professor Hagay Sobol, INSERM E9939, Oncologie Génétique, Institut Paoli-Calmettes, 232 boulevard Sainte Marguerite, 13273 Marseille, Cedex 9, France. Tel: + 334912238 36;

Fax: + 334912238 57; E-mail: sobol@marseille.inserm.fr

Received 19 November 2001; revised 27 May 2002; accepted 11 June 2002
}

(HBC) is known to account for 5 to $10 \%$ of all types of breast cancer, and $84 \%$ of these hereditary forms are thought to be due to the BRCA1/2 genes. ${ }^{1,2}$ The lifetime risk of a BRCA1/2 mutation carrier developing breast or ovarian cancer ranges on average from $56^{3}$ to $87 \%,{ }^{4}$ and from $16^{3}$ to $63 \%,{ }^{5}$ respectively. Since the main strategies used to search for germline mutations are both timeconsuming and expensive and these mutations are not very frequent in the populations studied so far, genetic tests are 
usually carried out first on the family member with the highest probability of being a BRCA1/2 mutation carrier among those who are available. This means that these tests are usually performed on a patient with breast and/or ovarian cancer. Secondly, since these mutations occur throughout the coding sequence, it is necessary to analyse the complete gene. Once the family mutation has been identified, pre-symptomatic BRCA1/2 diagnosis can be carried out on other family members to analyse the specific region of the gene carrying the mutation.

Presently in the United States, patents have been granted to a company that has opted for testing the BRCA1/2 genes by directly sequencing the genomic DNA (DS) of both genes. The same company has applied for the same patents in Europe. To date three of these patents have been examined and accepted by the European patent office (http://www.european-patent-office.org). Alternative strategies involving the use of a pre-screening stage to scan the entire gene and detect any variants, thus reducing the region of the gene requiring further characterisation by DS, have been devised and tested, however, by various research and hospital laboratories all over the world.

We carried out cost-effectiveness comparisons between the main alternative strategies available for detecting BRCA1/2 mutations and the DS method applied to the entire gene. Since these analytical techniques are similar in the case of both genes, the present study focused on the diagnosis of BRCA1 mutations alone as a model.

\section{Methods}

\section{Strategies for detecting BRCA1 gene mutations}

Alternative strategies (consisting of various combinations of techniques) were compared with DS as a means of searching the entire BRCA1 gene for point mutations, excluding large genetic alterations, by analysing the polymerase chain reaction (PCR) products obtained from the genomic DNA (gDNA) of an index case belonging to a high risk family. In the present study, we focused on the type of strategy, but not the mode of organisation of the laboratory conducting the analysis. We therefore assumed that the equipment available at the laboratories could be used to analyse only one set of samples at a time. Consequently, the laboratories were assumed to have no special battery of equipment. We focused on routine analysis and excluded large genetic alterations, the exact prevalence of which is still unknown but generally assumed to rate low, and which are at present being dealt with using other techniques, mostly in a research context. For specific populations where the prevalence of large genetic alterations is taken to be higher, particularly in case of founder effect, ${ }^{6}$ local organisation of BRCA analysis could integrate such techniques. Due to the fragility of messenger RNA (mRNA) and the differences which existed between the conditions of blood sample collection, transport, and storage, we included only techniques based on
gDNA. All the alternative strategies tested were based on a two-step process consisting first of rapidly scanning part or all of the gene, using pre-screening techniques to detect any anomalies (variants in the coding sequence). When any such anomalies were detected, a second step was carried out, in which the DS was performed on both strands of the region of interest. Six different candidate techniques for performing the pre-screening step were examined: denaturing high performance liquid chromatography (DHPLC), ${ }^{7}$ single-strand conformation polymorphism (SSCP), ${ }^{8}$ denaturing gradient gel electrophoresis (DGGE), ${ }^{9,10}$ heteroduplex analysis (HA), ${ }^{11}$ fluorescent assisted mismatch analysis (FAMA) ${ }^{12,13}$ and the protein truncation test (PTT). ${ }^{14-16}$

The BRCA1 gene is composed of 22 coding exons, one of which (exon 11) corresponds to $60 \%$ of the whole coding sequence. Apart from PTT, all the techniques, including DS of the entire gene, were used to investigate these 22 regions. PTT is generally applied only to analyse exon 11, because with this technique, it is preferable to use complementary DNA (cDNA) obtained from the mRNA to test the smaller exons, and because it is not suitable for identifying missense mutations that are taken to be deleterious, such as those involving the ring finger motif comprising exons 2 to 5. With all these techniques, the gene has to be broken up into fragments and with all of them except for PTT, the 21 fragments corresponding to the 21 small exons of the BRCA1 gene have to be examined successively. When it comes to analysing the large exon 11, however, the techniques differ as to the number of fragments required: some of them (DS, DHPLC, SSCP and DGGE) require a large number of small fragments $(n=14)$, while others require only a few larger fragments ( $n=4$ in the case of FAMA and PTT; $n=6$ in that of HA). Therefore, in addition to the five strategies consisting of applying the five pre-screening techniques to the entire gene (except for PTT), and then possibly applying DS to the specific regions in which variants have been detected, we tested 14 other strategies based on various combinations of HA, FAMA or PTT to screen exon 11, applying other techniques to the remaining 21 exons. The use of a different technique on exon 11 in the pre-screening step was envisaged only if this reduced the number of fragments screened. Details of the 20 strategies tested are given in Table 1.

In our assessment, all 20 strategies used were required to include the complete genetic testing process, from the receipt of the patient's blood sample to the diagnosis. Whenever a deleterious mutation was identified using any of the 20 strategies, a second blood sample from the same patient was analysed in order to confirm the diagnosis. In the second test, only DS had to be applied to the previously identified abnormal fragments.

The 20 BRCA1 mutation detection strategies were compared after testing them on a 'theoretical' population sample ( $n=10000$ individuals) with a $15 \%$ risk of harbouring 
Table 1 Strategies for performing a mutation search on the entire BRCA1 gene sequence

\begin{tabular}{|c|c|c|}
\hline \multicolumn{3}{|c|}{ Strategy ${ }^{a}$} \\
\hline$S_{1}$ & \multicolumn{2}{|l|}{ DS } \\
\hline $\mathrm{S}_{2}$ & DHPLC & $\rightarrow \mathrm{DS}_{\mathrm{F}}$ if a variant is detected \\
\hline $\mathrm{S}_{3}$ & SSCP & $\rightarrow \mathrm{DS}_{\mathrm{F}}$ if a variant is detected \\
\hline $\mathrm{S}_{4}$ & DGGE & $\rightarrow D S_{F}$ if a variant is detected \\
\hline $\mathrm{S}_{5}$ & $\mathrm{HA}$ & $\rightarrow \mathrm{DS}_{\mathrm{F}}$ if a variant is detected \\
\hline$S_{6}$ & FAMA & $\rightarrow \mathrm{DS}_{\mathrm{F}}$ if a variant is detected \\
\hline $\mathrm{S}_{7}^{\mathrm{b}}$ & $\mathrm{HA}_{11}+\mathrm{DS}_{21}$ & $\rightarrow \mathrm{DS}_{\mathrm{F}}$ if a variant is detected on exon 11 \\
\hline$S_{8}$ & $\mathrm{HA}_{11}+\mathrm{DHPLC}_{21}$ & $\rightarrow \mathrm{DS}_{\mathrm{F}}$ if a variant is detected \\
\hline $\mathrm{S}_{9}$ & $\mathrm{HA}_{11}+\mathrm{SSCP}_{21}$ & $\rightarrow \mathrm{DS}_{\mathrm{F}}$ if a variant is detected \\
\hline$S_{10}$ & $\mathrm{HA}_{11}+\mathrm{DGGE}_{21}$ & $\rightarrow \mathrm{DS}_{\mathrm{F}}$ if a variant is detected \\
\hline$S_{11}$ & $\mathrm{FAMA}_{11}+\mathrm{DS}_{21}$ & $\rightarrow D S_{F}$ if a variant is detected on exon 11 \\
\hline$S_{12}$ & $\mathrm{FAMA}_{11}+\mathrm{DHPLC}_{21}$ & $\rightarrow \mathrm{DS}_{\mathrm{F}}$ if a variant is detected \\
\hline $\mathrm{S}_{13}$ & FAMA $_{11}+$ SSCP $_{21}$ & $\rightarrow \mathrm{DS}_{\mathrm{F}}$ if a variant is detected \\
\hline $\mathrm{S}_{14}$ & $\mathrm{FAMA}_{11}+\mathrm{DGGE}_{21}$ & $\rightarrow \mathrm{DS}_{\mathrm{F}}$ if a variant is detected \\
\hline $\mathrm{S}_{15}$ & $\mathrm{FAMA}_{11}+\mathrm{HA}_{21}$ & $\rightarrow \mathrm{DS}_{\mathrm{F}}$ if a variant is detected \\
\hline$S_{16}$ & $\mathrm{PTT}_{11}+\mathrm{DS}_{21}$ & $\rightarrow \mathrm{DS}_{\mathrm{F}}$ if a variant is detected on exon 11 \\
\hline $\mathrm{S}_{17}$ & $\mathrm{PTT}_{11}+\mathrm{DHPLC}_{21}$ & $\rightarrow \mathrm{DS}_{\mathrm{F}}$ if a variant is detected \\
\hline $\mathrm{S}_{18}$ & $\mathrm{PTT}_{11}+\mathrm{SSCP}_{21}$ & $\rightarrow \mathrm{DS}_{\mathrm{F}}$ if a variant is detected \\
\hline $\mathrm{S}_{19}$ & $\mathrm{PTT}_{11}+\mathrm{DGGE}_{21}$ & $\rightarrow \mathrm{DS}_{\mathrm{F}}$ if a variant is detected \\
\hline$\underline{\mathrm{S}_{20}}$ & $\mathrm{PTT}_{11}+\mathrm{HA}_{21}$ & $\rightarrow \mathrm{DS}_{\mathrm{F}}$ if a variant is detected \\
\hline \multicolumn{3}{|c|}{ 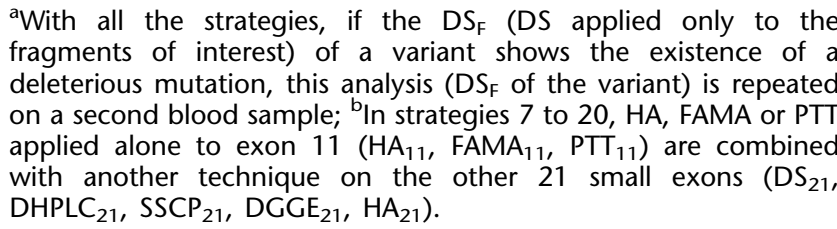 } \\
\hline
\end{tabular}

a deleterious mutation. This $15 \%$ probability level is that corresponding to a woman with breast cancer who has two first degree relatives with breast cancer; ${ }^{17}$ it also corresponds to the average frequency of the deleterious mutations observed in clinical series of breast cancer patients tested for BRCA1. ${ }^{18}$ We performed a sensitivity analysis, however, using alternative hypotheses from the literature as to the frequency of deleterious BRCA1 mutations in the 'theoretical' population tested, ranging from $2.5 \%$ (a general population of breast cancer patients without any additional selection criteria) ${ }^{19}$ to $30 \%$ (patients selected on the basis of early age at cancer onset and the morphological profile of the tumour) ${ }^{20}$ or $40 \%$ (breast cancer patients with a history of both breast and ovarian cancer in the family). ${ }^{17}$ Based on the data available on the deleterious BRCA1/2 mutations (http://www.nhgri.nih.gov/ Intramural_research/Lab_transfer/Bic), we also adopted the hypothesis that $50 \%$ of the deleterious mutations were likely to be detected in exon 11 and the other half in the remaining 21 exons.

\section{The effectiveness criterion}

The total number of deleterious mutations diagnosed in the population tested using a given strategy was used as the outcome variable.

Gold standard DS was taken to be the 'gold standard'. It is assumed that DS application to the entire gene would lead to a 100\% detection rate (no false negatives) as far as substitution mutations (deleterious missense or nonsense mutations, unknown variants, and polymorphisms) and small deletions or insertions were concerned. Because DS is not technically adapted to identify large genetic alterations, the detection of such mutations was therefore not considered in the present study. In addition, because only genetic alterations leading to truncated proteins and missense mutations in the ring finger domain are currently considered as disease associated mutations, it is assumed for the present study that DS does not induce false positive.

Other techniques The sensitivity values used for the other techniques were based on the literature and are presented in Table 2. The analysis was performed, first, using the mean sensitivity value, but was also carried out taking the maximum and minimum values published in the literature. About FAMA, although the literature gave a very high sensitivity rate $(100 \%)$, because these results are based on only two studies, ${ }^{13,31}$ we decided to evaluate the effect of decreasing the sensitivity on the cost-effectiveness result. Thresholds of sensitivity, corresponding to the elimination of the strategies using FAMA, were then identified.

The false negative rate of each strategy depends only on the sensitivity of the pre-screening techniques since DS focused on a fragment containing a possible anomaly cannot induce false negatives other than large genetic alterations and possible misinterpretations of sequence analysis. However these limitations are shared by all the strategies using DS. The false positives of every prescreening techniques are of two types: missense mutations of unknown significance and polymorphisms. Their subsequent characterisation is then checked by direct sequencing of the specific region of interest. Therefore we assumed that no false positives remained after DS.

Additional assumptions were necessary to be able to calculate how many fragments identified with the prescreening techniques justified subsequent DS analysis. In the case of PTT, these additional assumptions were not necessary, however, since only protein-truncating mutations can be detected with this technique. The probability of missense mutations of unknown significance being identified with the pre-screening methods but not being subsequently found upon DS analysis of the gene fragment to constitute deleterious mutations worked out at $3 \%$, based on the data in the literature. ${ }^{19,33}$ Concerning the checking of polymorphism, in a sample of 49 breast cancer patients tested at the Paoli-Calmettes Institute, only 34 out of the 118 cases of polymorphism observed necessitated a DS analysis because the recurrence and the profiles of several of these variants suggested that they were not associated with deleterious values. So we considered that 0.7 polymorphism per individual tested had to be checked by DS of the area of interest. A sensitivity analysis was 
Table 2 Technical characteristics of the various techniques

\begin{tabular}{|c|c|c|c|c|c|c|}
\hline \multirow[b]{2}{*}{ Techniques } & \multicolumn{3}{|c|}{ Number of fragments per BRCA1 gene } & \multirow{2}{*}{$\begin{array}{l}\text { Maximum number of fragments } \\
\text { screened per year }{ }^{a}\end{array}$} & \multicolumn{2}{|c|}{ Sensitivity values per fragment ${ }^{b}$} \\
\hline & Exon11 & Rest & Total & & Range & References \\
\hline DS & 14 & 21 & 35 & $7378\left(86^{\mathrm{c}}\right)$ & $100 \%$ & Gold standard \\
\hline DHPLC & 14 & 21 & 35 & $25606\left(298^{\mathrm{C}}\right)$ & {$[92 \% ; 100 \%]$} & $21-26$ \\
\hline SSCP & 14 & 21 & 35 & $10416\left(121^{\mathrm{c}}\right)$ & {$[60 \% ; 100 \%]$} & $22,24,26-2 \varepsilon$ \\
\hline DGGE & 14 & 21 & 35 & $24304\left(276^{\mathrm{c}}\right)$ & {$[80 \% ; 100 \%]$} & 29,30 \\
\hline $\mathrm{HA}$ & 6 & 21 & 27 & $29295\left(473^{c}\right)$ & {$[74 \% ; 89 \%]$} & 24 \\
\hline FAMA & 4 & 21 & 25 & $6076\left(124^{c}\right)$ & $100 \%$ & 13,31 \\
\hline PTT & 4 & - & - & $11935\left(^{d}\right)$ & $92 \%{ }^{\mathrm{e}}$ & 32 \\
\hline
\end{tabular}

${ }^{a}$ Corresponding to the $100 \%$ activity of the specific equipment required for each technique; ${ }^{b}$ We referred here only to published studies fulfilling the following criteria: (1) they had to include comparisons between the pre-screening technique and DS, (2) optimum technical conditions for applying the technique had to be used, (3) in those cases where several studies were available, those in which the technique was applied to the largest number of fragments were selected; ${ }^{C}$ Equivalent in terms of the number of patients screened for BRCA1/2 calculated on the basis of the number of fragments screened with each technique for the BRCA1 gene and for the BRCA2 gene, given that DS, DHPLC, SSCP,

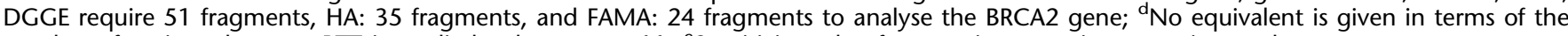
number of patients because PTT is applied only to exon 11; 'Sensitivity value for protein-truncating mutations only.

performed, however, in which every possible polymorphism detected by a pre-screening technique was analysed by performing DS.

\section{Cost assessment}

The direct costs were assessed on the basis of detailed studies carried out at three different laboratories (the Curie Institute, the Gustave Roussy Institute and the Paoli-Calmettes Institute), all of which use DS and DHPLC, and some of the other techniques (PTT, FAMA at the first laboratory; SSCP, HA at the second one; and DGGE at the third one). The costs were assessed by measuring the physical quantities of consumable supplies, equipment and labour (in terms of time spent by the laboratory staff) used in the case of each strategy. Monetary values were assigned to these quantities on the basis of the average 2002 prices (in Euros) obtaining at the three laboratories which participated in the study.

The following assumptions were made in estimating the cost per DNA fragment analysed with each technique. Each type of equipment was assumed to be used to full capacity (100\%) on an annual basis, and the labour force was assumed to be optimally productive during the number of working days per year $(n=217)$ imposed by the French legislation. According to these hypotheses, the maximum number of fragments that could be screened per year per item of appropriate equipment is indicated in Table 2. A sensitivity analysis was also performed, however, using alternative rates (10 to $90 \%$ ) as far as the use of equipment was concerned. The annual cost of each piece of equipment was calculated on the basis of a $20 \%$ depreciation rate and a $8 \%$ discount rate. With each strategy, the cost of preparing the blood sample upon receipt and that of the usual procedure used to prepare gDNA from the blood samples was also included, but the unit costs of these operations were identical with all the strategies examined. A different unit cost of gDNA preparation was then used in the case of DS confirmation of the diagnosis of a deleterious mutation on a second blood sample, since faster techniques can be used in this case.

\section{Cost-effectiveness analysis}

An average cost per deleterious mutation detected in the population tested was calculated for each strategy. Dominated strategies (ie, strategies where there existed at least one alternative strategy giving an equal or greater total number of detected mutations and a lower average cost per mutation detected) were identified. ${ }^{34}$ The increase in the cost-effectiveness ratios (the additional cost per additional mutation detected when switching from one strategy to another) was also calculated between the remaining optimum strategies.

\section{Results}

\section{Cost of the strategies}

Table 3 gives details of the unit cost obtained per DNA fragment analysed with each technique. Not surprisingly, all the pre-screening techniques, with the exception of FAMA, were found to have lower unit costs than DS. Most of the pre-screening techniques are labour intensive, and only a small proportion $(<10 \%)$ of their unit cost goes to the depreciation of the equipment. However, the cost of equipment accounts for a large proportion of the unit cost in the case of DHPLC and to a lesser extent, FAMA and DS. In addition, the usual preliminary stages were assessed at $8 €$ per blood sample, including the cost of preparing the blood samples upon receipt and performing the usual gDNA preparation procedure and $9.3 €$ when a faster method of gDNA preparation was used.

As the techniques differed as regards the number of fragments they were designed to analyse and the number of variants that would require a DS characterisation step, comparing the costs really makes sense only if the complete analysis of the BRCA1 gene in a given individual is taken as the basis of the comparisons. In Table 4, the twenty strategies are ranked in increasing order of absolute effectiveness 
Table 3 Cost per fragment with each technique, giving the distribution between cost factors (2002 Euros $€$ )

\begin{tabular}{|c|c|c|c|c|c|c|c|}
\hline Cost factor & $D S$ & $D H P L C$ & SSCP & $D G G E$ & $H A$ & FAMA & PTT \\
\hline Equipment & $5.2 €(18)$ & $1.6 €(41)$ & $0.3 €(5)$ & $0.2 €(4)$ & $0.1 €(3)$ & $6.7 €(25)$ & $0.2 €(2)$ \\
\hline
\end{tabular}

Figures in brackets are percentages of the total cost.

(total number of mutations detected in a theoretical population of 10000 patients with a $15 \%$ probability of deleterious mutation). The 'gold standard' strategy, involving the use of DS on the entire BRCA1 gene $\left(S_{1}\right)$, is logically that with the highest absolute effectiveness, but Table 4 shows that it is also associated with the highest total costs and the highest average cost of diagnosis per patient. By comparison, strategies in which pre-screening techniques are used can reduce the total costs of diagnosis by $30 \%\left(\mathrm{~S}_{11}\right)$ to nearly $90 \%\left(\mathrm{~S}_{5}\right)$. In most cases, this cost reduction is associated, however, with a loss of absolute effectiveness, but it is worth noting that two strategies $\left(\mathrm{S}_{6}\right.$ and $S_{11}$ ) in which FAMA is used as the pre-screening technique can reach similar levels of effectiveness to that which can be achieved with DS of the entire gene, while costing less.

Figure 1 shows the impact of alternative hypotheses as to the annual rate of use of equipment on the average cost of diagnosis per patient. This figure confirms that DS of the entire gene is always the most expensive strategy, regardless of the rate of use of the equipment. It shows that the average cost per diagnosis starts to increase significantly at a quite low rate of use $(<20 \%)$. As was to be expected in view of the unit cost of analysis per fragment (Table 3), the only exceptions are the strategies in which DHPLC is used as the pre-screening technique (see strategy $\mathrm{S}_{2}$ in Figure 1), where the average cost per diagnosis increases sharply as soon as the equipment is used at a rate of less than $40 \%$.

\section{Cost-effectiveness analysis}

The most cost-effective strategy $\left(\mathrm{S}_{20}\right)$ was found to be that in which PTT was used to screen exon 11 and HA to screen the other 21 exons (Table 4). In a population with a $15 \%$ prevalence of deleterious BRCA1 mutations, however, this strategy would yield a large number of false negatives (13\%). Other strategies involving a pre-screening stage may nevertheless be worthwhile, although they are associated with less favourable cost-effectiveness ratios because they yield fewer false negatives than $S_{20}$ : this is so in the case of the strategies involving either a combination between PTT and DHPLC $\left(\mathrm{S}_{17}\right)$ or DHPLC alone $\left(\mathrm{S}_{2}\right)$, and those involving either a combination between FAMA and DHPLC $\left(\mathrm{S}_{12}\right)$ or FAMA alone $\left(\mathrm{S}_{6}\right)$ which were used to screen the gene before DS was applied to the regions in which any variants had been identified.

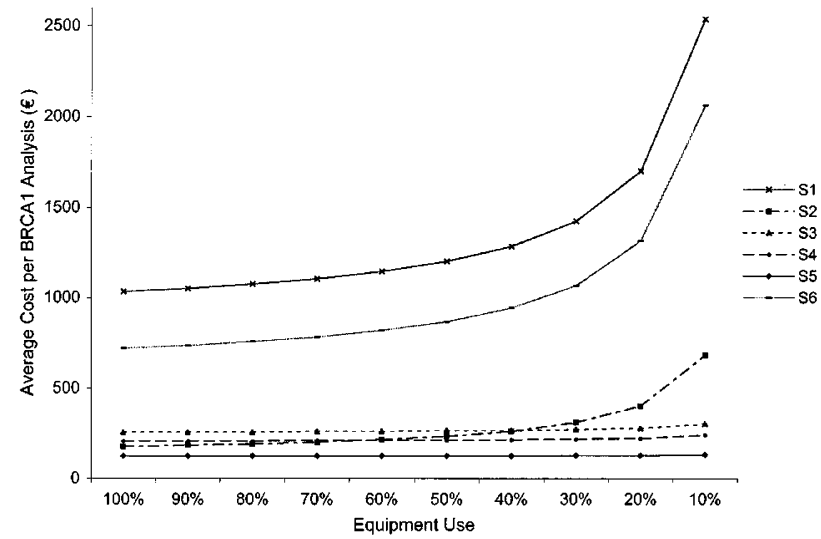

Figure 1 Effects of the use of special equipment on the average cost per analysis of the gene.

Consequently, DS of the entire gene is a strategy $\left(S_{1}\right)$ which was found here (Table 4 ) to be completely dominated by at least one alternative strategy $\left(\mathrm{S}_{6}\right)$ : screening the gene with FAMA before applying DS also scored $100 \%$ in terms of effectiveness, while being a less costly and therefore more cost-effective strategy. It can also be seen from Table 4 that such absolute total effectiveness can only be reached at the expense of sharply increasing costs. Switching from $\mathrm{S}_{12}$ (FAMA for screening exon $11+$ DHPLC for the 21 other exons) to $S_{6}$ (FAMA for screening the entire gene) would entail an increase in the cost per additional mutation detected in this population of about $163000 €$.

When the sensitivity analysis was performed with alternative hypotheses as to the prevalence of deleterious mutations (from 2.5 to $40.0 \%$ ) in the target population, the same cost-effectiveness hierarchy between strategies was obtained. However, the increase in the costs accruing per additional mutation detected between the optimum strategies tended to be lower in the case of populations where the prevalence of deleterious mutations was greater than 15\%; in a very high risk population (one with a $40 \%$ rate of occurrence of deleterious mutations), the increase in the cost per additional mutation would be as follows: $745 €$ when switching from $S_{20}$ to $S_{17}, 3668 €$ from $S_{17}$ to $S_{2}, 6845 €$ from $S_{2}$ to $S_{12}$ and $61232 €$ from $S_{12}$ to $S_{6}$.

Using other hypotheses as to the costs, like the discount rates and variations in the number of poly- 
Table 4 Cost and effectiveness of the various available strategies for performing a mutation search on the BRCA1 gene ordered by growing effectiveness for a 'theoretical' population of 10000 patients with a $15 \%$ probability of deleterious BRCA1 mutation (2002 Euros $€$ )

\begin{tabular}{|c|c|c|c|c|c|}
\hline Strategy & Total cost & $\begin{array}{l}\text { Total number of } \\
\text { mutations detected }\end{array}$ & $\begin{array}{l}\text { Average cost per } \\
\text { patient analysed }\end{array}$ & $\begin{array}{l}\text { Average cost per } \\
\text { mutation detected }\end{array}$ & $\begin{array}{c}\text { Additional cost per additiona } \\
\text { mutation detected }\end{array}$ \\
\hline$S_{3}$ & 2541684 & 1200 & 254.2 & 2118.1 & $-{ }^{a}$ \\
\hline$S_{9}$ & 1880212 & 1215 & 188.0 & 1547.5 & $-{ }^{a}$ \\
\hline $\mathrm{S}_{5}$ & 1209312 & 1230 & 120.9 & 983.2 & $-{ }^{a}$ \\
\hline$S_{10}$ & 1528711 & 1290 & 152.9 & 1185.1 & $-{ }^{a}$ \\
\hline $\mathrm{S}_{18}$ & 2684029 & 1290 & 268.4 & 2080.6 & $-{ }^{a}$ \\
\hline $\mathrm{S}_{20}\left(\mathrm{PTT}_{11}+\mathrm{HA}_{21} \rightarrow \mathrm{DS}_{\mathrm{F}}\right)$ & 1267517 & 1305 & 126.8 & 971.3 & 971.3 \\
\hline $\mathrm{S}_{8}$ & 1406010 & 1335 & 140.6 & 1053.2 & $-{ }^{a}$ \\
\hline $\mathrm{S}_{4}$ & 2047682 & 1350 & 204.8 & 1516.8 & $-{ }^{a}$ \\
\hline $\mathrm{S}_{13}$ & 2772682 & 1350 & 277.3 & 2053.8 & $-{ }^{a}$ \\
\hline $\mathrm{S}_{19}$ & 1586916 & 1365 & 158.7 & 1162.6 & $-{ }^{a}$ \\
\hline$S_{15}$ & 2101782 & 1365 & 210.2 & 1539.8 & $-{ }^{a}$ \\
\hline$S_{7}$ & 6572170 & 1365 & 657.2 & 4814.8 & $-{ }^{a}$ \\
\hline $\mathrm{S}_{17}\left(\mathrm{PTT}_{11}+\mathrm{DHPLC}_{21} \rightarrow \mathrm{DS}_{\mathrm{F}}\right)$ & 1464215 & 1410 & 146.4 & 1038.5 & 1873.3 \\
\hline $\mathrm{S}_{14}$ & 2421181 & 1425 & 242.1 & 1699.1 & $-{ }^{a}$ \\
\hline $\mathrm{S}_{2}\left(\mathrm{DHPLC} \rightarrow \mathrm{DS}_{\mathrm{F}}\right)$ & 1754281 & 1440 & 175.4 & 1218.3 & 9668.9 \\
\hline$S_{16}$ & 6630375 & 1440 & 663.0 & 4604.4 & $-{ }^{a}$ \\
\hline $\mathrm{S}_{12}\left(\mathrm{FAMA}_{11}+\mathrm{DHPLC}_{21} \rightarrow \mathrm{DS}_{\mathrm{F}}\right)$ & 2298480 & 1470 & 229.9 & 1563.6 & 18140.0 \\
\hline $\mathrm{S}_{6}\left(\mathrm{FAMA} \rightarrow \mathrm{DS}_{\mathrm{F}}\right)$ & 7193680 & 1500 & 719.4 & 4795.8 & 163173.3 \\
\hline $\mathrm{S}_{11}$ & 7464640 & 1500 & 746.5 & 4976.4 & $--^{a}$ \\
\hline$S_{1}(D S)$ & 10322600 & 1500 & 1032.3 & 6881.7 & $-{ }^{a}$ \\
\hline
\end{tabular}

${ }^{\text {a }}$ Strategies that are dominated.

morphisms analysed by DS, did not affect the results of the sensitivity analysis. Strategies $S_{20}$ and $S_{12}$ remain optimum whatever the use level of the equipment is for all techniques. Strategies $S_{17}$ and $S_{2}$ are not dominated as far as the use of the specific equipment for DHPLC is at least 40 and 70\%, respectively, regardless the use level of the equipment for the other techniques. Finally, $\mathrm{S}_{6}$ remains optimum until the specific equipment for FAMA and DS is used at least at 50\%, whatever the activity is for the other techniques.

Using various hypothetical sensitivity values to indicate the detection rates of the various techniques (Table 2) has affected the results in some extreme cases. If the rate of deleterious mutations in exon 11 detected by PTT is taken to be lower than $85 \%$, strategies $S_{20}$ and $S_{17}$ have to be eliminated from the optimum strategies. If DHPLC, SSCP or DGGE are taken to be capable of detecting $100 \%$ of the deleterious mutations (the highest estimated sensitivity given in the literature), the strategy $S_{6}$ in which FAMA is used will be dominated, and $\mathrm{S}_{2}, \mathrm{~S}_{3}$ or $\mathrm{S}_{4}$ will rank higher than DS of the entire gene. In the framework of this assumption, the strategies in which either $S_{2}, S_{3}$ or $S_{4}$ is used for pre-screening purposes therefore rank as high as DS on the entire gene in terms of their effectiveness, while costing less. Because the reported high sensitivity of FAMA is based on few studies, we have analysed the impact of the variation of this parameter. As far as FAMA was not associated to a $100 \%$ sensitivity, $\mathrm{S}_{6}$ was excluded from the optimum strategies and DS $\left(\mathrm{S}_{1}\right)$ could be considered as an optimum solution. The strategies involving FAMA as a pre-screening technique for the exon 11 associated to
DHPLC $\left(\mathrm{S}_{12}\right)$ or DS $\left(\mathrm{S}_{11}\right)$ were optimum until the sensitivity of FAMA reached the one of DHPLC (96\%) since this technique was less expensive.

\section{Discussion}

Surprisingly, to date, only a few economic analyses have been carried out in the field of genetic breast cancer predisposition. ${ }^{35,36}$ To our knowledge, the present study is the first in which it has been attempted to apply costeffectiveness analysis to compare various strategies for DNA testing of breast cancer predisposing genes.

Our analysis shows that the cost of BRCA1 diagnosis on an index case using DS including the confirmation on a second blood sample of a deleterious mutation if identified is very high (1032€ $\approx 896$ US\$ for BRCA1 only). In contrast, the cost of additional BRCA1 testing on other family members is lower $(76.8 € \approx 66.7$ US $\$)$. Importantly, we demonstrate that there exist alternative strategies for performing BRCA1 diagnosis: pre-screening techniques such as FAMA and potentially, DHPLC, SSCP or DGGE, based on the current estimates of their sensitivity, would minimise the cost of diagnosis while also ensuring a comparable level of effectiveness to that of applying DS to the entire gene. Our results show that other strategies involving a pre-screening stage could be even more cost-effective (ie reducing three or fivefold the average cost per deleterious mutation detected in a population with a $15 \%$ prevalence), as long as a higher false negative rate (ranging from 2 to 13\%) is deemed to be acceptable. Conversely, it can also be considered that a test which is only performed once in the lifetime, 
with major health implications for the patient and his/her family, the probability of detecting a mutation when it is actually present (sensitivity) is a crucial parameter. When deciding about the financial coverage of a particular strategy, health policy makers should therefore pay much attention to the final consequences in terms of medical management of women when a mutation is identified and also taking into account that given the sensitivity of various strategies, false negatives may exist. Here the economic evaluation included only the cost and the effectiveness of identifying a first mutation in a family. The preventive management for mutation carriers, studied previously, ${ }^{35,37,38}$ and the consequence of the lack of specific follow up in false negatives were excluded from our analysis.

The present assessment admittedly has some limitations. First, our detailed analysis of the items consumed when using alternative strategies to perform BRCA1 mutation searches was carried out at three laboratories in a single country (France). Further cost studies at additional laboratories operating in different countries are now required to confirm the validity of our estimates. However, the techniques under study are based on standard procedures and products and the respective costs do not seem likely to vary greatly from one country to another in the Western world. Secondly, our ratings of the effectiveness of the strategies selected were based on the best evidence available in the literature at the time of the study. But since these techniques are still being improved, their effectiveness will probably increase in the future. However, we performed an extensive sensitivity study on all the parameters liable to affect the results of our cost-effectiveness analysis. The results suggest that our conclusions are unlikely to be shaken by additional data, unless further improvements in the techniques make it possible to achieve economies of scale and decrease the resulting cost of mutation search. It is possible that DS may be improved by using for example a 96 capillary format and the latest software programs to analyse the sequence data. A similar breakthrough is also foreseeable in the case of the other automated techniques selected here as being optimum strategies (DHPLC, FAMA), whereas no such advances have yet been made as far as the other techniques (HA, PTT) are concerned. Thirdly, since we proposed to analyse the performances of the various strategies but not the mode of organisation used at the laboratories, no battery of equipment such as that which might be expected to exist at the industrial level was assumed to be available. New breakthroughs and/or new modes of organisation can naturally be expected to reduce the costs and the productivity of all DS, DHPLC and FAMA, but this will not affect the hierarchy of optimum strategies in any way. The organisation of BRCA testing in an industrial context would mainly favour rapid delivery, but would also involve higher initial investments for the test providers.
Rapid service is a priority for the follow up of patients with a high genetic risk, especially if they subsequently have to undergo prophylactic surgery. In the context of automated strategies, the time required to obtain results depends first on the time taken to collect the number of individuals corresponding to the full capacity of the equipment before starting the analysis and secondly, on the time taken to complete the analysis on the set of individuals. It is therefore possible to reduce the 'time' factor by reducing both components. The time required to collect the right number of individuals depends on the management of the recruitment area, whereas the most time-saving factor in the analysis is not the type of strategy, but rather the mode of organisation, and it will therefore be necessary to subsequently opt for a battery of equipment capable of running in parallel and thus of coping flexibly with the ongoing demand.

It is a well-established fact in various fields where genetic screening is practised that charging high direct costs to patients may constitute a major barrier preventing people from having access to these testing procedures, ${ }^{39}$ and that health care insurance coverage, whether it is publicly or privately funded, may greatly facilitate access to these diagnostic techniques. Insufficient insurance coverage has already been identified as one of the main causes of the slowness with which BRCA1/2 diagnosis is being adopted in the United States (http://www.geneletter.com/04-01-00/ features/breastcancer.html). ${ }^{40}$ If the requirements of patent owners make it impossible to use the most cost-effective strategies, this may well lead health insurance systems elsewhere (especially in Europe, where governmental efforts are being made to control the escalation of health care expenditure) to delay the inclusion of breast cancer susceptibility genetic testing in their package, even in the case of the high-risk women who are the most in need of this diagnosis. ${ }^{41}$ Therefore a rational utilisation of health insurance funds require that a comparative assessment of cost-effectiveness of different diagnostic strategies should be analysed before the process of reimbursement be definitely adopted. Our study is the first contribution in this field.

\footnotetext{
Acknowledgements

We thank Virgine Caux, Anthony Laugé and Sabine Pagès from the Curie Institute, Michel Barrois, Johny Bombled, Stéphane Jankowski and Antoine Piriou from the Gustave Roussy Institute, and José Adélaïde, Florence Allione, Fabienne Kerangueven and Véronique Wargniez from the Paoli-Calmettes Institute for their technical assistance. We thank Olga Sinilnikova from the Edouard Herriot Hospital and the International Agency for Cancer Research, Mario Tosi from the Pasteur Institute, and Daniel Birnbaum, Tetsuro Noguchi and Richard Sauvan from the Paoli-Calmettes Institute for helpful discussions, advice and technical comments. This study was supported by the French National Institute for Health and Medical Research (INSERM), the Paoli-Calmettes Institute and by grants from La Ligue Contre le Cancer and the Association pour la Recherche sur le Cancer (ARC).
} 


\section{References}

1 Ford D, Easton DF, Stratton $\mathrm{M}$ et al: Genetic heterogeneity and penetrance analysis of the BRCA1 and BRCA2 genes in breast cancer families. Am J Hum Genet 1998; 62: 676-689.

2 Eisinger F, Alby N, Bremond A et al: Recommendations for medical management of hereditary breast and ovarian cancer: the French National Ad Hoc Committee. Ann Oncol 1998; 9: $939-$ 950.

3 Struewing JP, Hartge P, Wacholder S et al: The risk of cancer associated with specific mutations of BRCA1 and BRCA2 among Ashkenazi Jews. N Engl J Med 1997; 336: 1401-1408.

4 Ford D, Easton DF, Bishop DT, Narod SA, Golfgar DE: Breast Cancer Linkage Consortium: Risks of cancer in BRCA1-mutation carriers. Lancet 1994; 343: 692-695.

5 Easton D, Narod S, Ford D, Steel M, on the behalf of the Breast Cancer Linkage Consortium: The genetic epidemiology of BRCA1. Lancet 1994; 344: 761.

6 Petrij-Bosch A, Peelen T, van Vliet M et al: BRCA1 genomic deletions are major founder mutations in Dutch breast cancer patients. Nat Genet 1997; 17: 341-345.

7 Oefner PJ, Underhill PA: Comparative DNA sequencing by denaturing high performance chromatography (DHPLC). Am J Hum Genet 1995; 57 (Suppl): A266.

8 Orita M, Iwahana H, Kanazawa H, Hayashi K, Seyita T: Detection of polymorphisms of human DNA by gel electrophoresis as single-strand conformation polymorphism. Proc Natl Acad Sci USA 1989; 86: 2766-2770.

9 Fischer SG, Lerman LS: DNA fragments differing by single basepair substitutions are segregated in denaturing gradient gel: correspondence with melting theory. Proc Natl Acad Sci USA 1983; 80: $1579-1583$.

10 Sheffield VC, Cox DR, Lerman LS, Myers RM: Attachment of a 40base-pair G+C-rich sequence (G-C clamp) to genomic DNA fragments by the polymerase chain reaction results in improved detection of single-base changes. Proc Natl Acad Sci USA 1989; 86: $232-236$.

11 White MB, Carvalho M, Derse D, O'Brien S, Dean M: Detecting single base substitutions as heteroduplex polymorphisms. Genomics 1992; 12: $301-306$.

12 Verpy E, Biasotto M, Meo T, Tosi M: Efficient detection of point mutations in color-coded strands of target DNA. Proc Natl Acad Sci USA 1994; 91: 1873-1877.

13 Verpy E, Biasotto M, Brai M, Misiano G, Meo T, Tosi M: Exhaustive mutation scanning by fluorescent-assisted mismatch analysis discloses new genotype-phenotype correlations in angiodema. Am J Hum Genet 1996; 59: 308-319.

14 Roest PAM, Roberts RG, Sugino S, van Ommen GJB, den Dunnen JT: Protein Truncation Test (PTT) for rapid detection of translation-terminating mutations. Hum Mol Genet 1993; 2: 1719-1721.

15 van der Luijt RB, Meera Kahn P, Vasen $\mathrm{H}$ et al: Rapid detection of translation-terminating mutations at the adenomatous polyposis coli (APC) gene by direct protein truncation test. Genomics 1994; 20: $1-4$.

16 Powell SM, Petersen GM, Krush AAJ et al: Molecular diagnosis of familial adenomatous polyposis. N Engl J Med 1993; 329: 19821987.

17 Stoppa-Lyonnet D, Laurent-Puig P, Essioux L et al: BRCA1 sequence variations in 160 individuals referred to a breast/ovarian family cancer clinic. Am J Hum Genet 1997; 60: 1021-1030.

18 Shattuck-Eidens D, Oliphant A, McClure M et al: BRCA1 sequence analysis in women at high risk for susceptibility mutations. Risk factor analysis and implications for genetic testing. JAMA 1997; 278: $1242-1250$.

19 Newman B, Mu H, Butler LM, Millikan RC, Moorman PG, King MC: Frequency of breast cancer attributable to BRCA1 in a population-based series of american women. JAMA 1998; 279: 915921.

20 Lidereau R, Eisinger F, Champeme MH et al: Major improvement in the efficacy of BRCA1 mutation screening using morphoclinical features of breast cancer. Cancer Res 2000; 60: 1206-1210.
21 Arnold N, Gross E, Schwarz-Boeger U, Pfisterer J, Jonat W, Kiechle M: A highly sensitive, fast and economical technique for mutation analysis. Hum Mutat 1999; 14: 333-339.

22 Gross E, Arnold N, Goette J, Schwarz-Boeger U, Kiechle M: A comparison of BRCA1 mutation analysis by direct sequencing, SSCP and DHPLC. Hum Genet 1999; 105: 72-78.

23 O'Donovan M, Oefner PJ, Roberts SC et al: Blind analysis of denaturing high performance liquid chromatography as a tool for mutation detection. Genomics 1998; 52: 44-49.

24 Jones AC, Austin J, Hansen N et al: Optimal temperature selection for mutation detection by denaturing HPLC and comparison to single-stranded conformation polymorphism and heteroduplex analysis. Clin Chem 1999; 45: 1133-1140.

25 Wagner T, Stoppa-Lyonnet D, Fleischmann E et al: Denaturinghigh performance liquid chromatography detects reliably BRCA1 and BRCA2 mutations. Genomics 1999; 62: 369-376.

26 Eng C, Brody LC, Wagner TMU et al: Interpreting epidemiological research: blinded comparison of methods used to estimate the prevalence of inherited mutations in BRCA1. J Med Genet 2001; 38: $824-833$.

27 Ravnik-Glavac M, Glavac D, Dean M: Sensitivity of single-strand conformation polymorphism and heteroduplex method for mutation detection in the cystic fibrosis gene. Hum Mol Genet 1994; 3: 801-807.

28 Ushijima T, Hosoya Y, Suzuki T, Sofuni T, Sugimura T, Nagao M: A rapid method for detection of mutations in the lac1 gene using PCR-single strand conformation polymorphism analysis: demonstration of its high sensitivity. Mutat Res 1995; 334: 283-292.

29 Gejman PV, Cao Q, Guedj F, Sommer S: The sensitivity of denaturing gradient gel electrophoresis: a blinded analysis. Mutat Res 1998; 382: $109-114$.

30 Miller KM, Ming TJ, Schulze AD, Withler RE: Denaturing gradient gel electrophoresis (DGGE): a rapid and sensitive technique to screen nucleotide sequence variation in populations. Biotechniques 1999; 27: 1016-1018, 1020-1022, 1024 passim.

31 Ricevuto E, Sobol H, Stoppa-Lyonnet D et al: Diagnostic strategy for analytical scanning of BRCA1 gene by fluorescence-assisted mismatch analysis using large bifluorescently labeled amplicons. Clin Cancer Res 2001; 7: 1638-1646.

32 Garvin AM: A complete protein truncation test for BRCA1 and BRCA2. Eur J Hum Genet 1998; 6: 226-234.

33 Garcia-Patino E, Gomendio B, Provencio $\mathrm{M}$ et al: Germline BRCA1 mutations in women with sporadic breast cancer: clinical correlations. J Clin Oncol 1998; 16: 115-120.

34 Weinstein MC: Principles of cost-effective resource allocation in health care organizations. Int J Technol Assess Health Care 1990; 6: $93-105$.

35 Grann VR, Whang W, Jacobson JS, Heitjan DF, Antman KH, Neugut AI: Benefits and costs of screening Ashkenazi Jewish women for BRCA1 and BRCA2. J Clin Oncol 1999; 17: 494-500.

36 Heimdal K, Maelhe L, Moller P: Costs and benefits of diagnosing familial breast cancer. Dis Markers 1999; 15: 167-173.

37 Schrag D, Kuntz KM, Garber JE, Weeks JC: Decision analysis Effects of prophylactic mastectomy and oophorectomy on life expectancy among women with BRCA1 or BRCA2 mutations. $N$ Engl J Med 1997; 336: 1465 - 1471.

38 Schrag D, Kuntz KM, Garber JE, Weeks JC: Life expectancy gains from cancer prevention strategies for women with breast cancer and BRCA1 or BRCA2 mutations. JAMA 2000; 283: 617-624.

39 Moatti JP, Le Gales C, Julian C, Durbec JP, Mattéi JF, Aymé S: Socio-cultural inequities in access to prenatal diagnosis: the role of insurance coverage and regulatory policies. Prenat Diagn 1990; 10: 313-325.

40 Lehrman S: Selling the breast cancer susceptibility test. GeneLetter 2000; 1.

41 Schoonmaker MM, Bernhardt BA, Holtzman NA: Factors influencing health insurers' decisions to cover new genetic technologies. Int J Technol Assess Health Care 2000; 16: 178-189. 